\title{
COPD und Studien
}

\section{Ergebnisse des Expertentreffens Luftschlösser 2010, Mainz, 3. -4. Dezember 2010 (Sponsor: Boehringer Ingelheim Pharma GmbH \& Co KG)}

\author{
COPD and Clinical Trials \\ Results of an Expert Meeting „Castles in the Air“ 2010
}

Autoren

Institute
J. Lorenz ${ }^{1}$, R. Bals ${ }^{2}$, A. Gillissen ${ }^{3}$, H. Magnussen ${ }^{4}$, M. Pfeifer ${ }^{5}$, W. Randerath ${ }^{6}$, G. Schultze-Werninghaus ${ }^{7}$, G. Steinkamp ${ }^{8}$, H. Teschler ${ }^{9}$, C. Vogelmeier ${ }^{10}$, H. Worth ${ }^{11}$

Die Institutsangaben sind am Ende des Beitrags gelistet.

\section{Bibliografie}

DOI http://dx.doi.org/

10.1055/s-0030-1256393

Online-Publikation: 8. 6. 2011

Pneumologie 2011; 65:

436-448 ๔ Georg Thieme

Verlag KG Stuttgart · New York ISSN 0934-8387

\section{Korrespondenzadresse}

Prof. Dr. med. Joachim Lorenz Klinik für Pneumologie und Internistische Intensivmedizin Klinikum Lüdenscheid Paulmannshöher Str. 14 58515 Lüdenscheid joachim.Iorenz@klinikumluedenscheid.de

\section{Zusammenfassung \\ $\nabla$}

Klinische Studien zur COPD haben zum Ziel, Fortschritte in der Diagnostik und Therapie der Erkrankung zu ermöglichen. Ihre Ergebnisse sollen für eine möglichst große Gruppe von Patienten anwendbar sein. Analysiert man aktuelle und ältere Studien genauer hinsichtlich ihrer Methodik und ihres Studiendesigns, werden Problembereiche sichtbar. Die COPD selbst ist eine komplexe Erkrankung mit unterschiedlichen Phänotypen. Genetische Grundlagen müssen systematischer untersucht werden, um eine Stratifizierung der Patienten zu ermöglichen. Vormals als robust eingeschätzte Zielparameter wie die FEV1 haben Grenzen, wie aktuelle Studien gezeigt haben. Dementsprechend wird nach neuen, prognoserelevanten Surrogat-Parametern gesucht, zu denen Komposit-Endpunkte und Biomarker gehören. Für die Beurteilung der Krankheitsprogression spielen körperliche Aktivität und Belastbarkeit eine zunehmende Rolle. Schwerpunkte der Medikamentenentwicklung sind lang wirksame Broncholytika und neue antiinflammatorische Substanzen. Zusätzlich wird der Wert nicht-medikamentöser Therapien evaluiert.

\section{Lässt sich die COPD in Studien pressen? $\nabla$}

\section{Referent: J. Lorenz}

\section{Phänotypen}

Bei der Planung und Ergebnisinterpretation klinischer Prüfungen geht man im Allgemeinen davon aus, dass der klinische Endpunkt oder der Surrogat-Marker normal verteilt ist und wie eine Gaußkurve nur einen Häufigkeitsgipfel aufweist. Für viele Parameter ist dies auch der Fall. Es gibt jedoch auch Endpunkte, deren Verteilung in der Population mehrere Häufigkeitsgipfel hat. Dies hängt mit der Komplexität der COPD zusammen.

\section{Abstract \\ $\nabla$}

Clinical trials in COPD patients aim at achieving progress in diagnosis and treatment. Study results should be applicable to a large number of patients. However, an analysis of the methods and design of current and previous trials reveals considerable room for improvement. COPD is a complex disease with different clinical phenotypes. Genetic factors need to be evaluated systematically to allow appropriate stratification of patients. Frequently used endpoints such as the FEV1 that had previously been considered reliable have shown limitations in recent trials. Thus, researchers now aim to identify new surrogate parameters that are related to the prognosis of the disease, e.g., composite endpoints and biomarkers. Physical activity and capacity are becoming increasingly important for the evaluation of disease progression. The focus of pharmaceutical development is long acting bronchodilators and new anti-inflammatory drugs. The value of non-drug interventions will also be evaluated.

Die Vielfältigkeit klinischer Ausprägungen und Phänotypen der COPD hat einen starken Einfluss auf die Ergebnisse klinischer Studien. Dies gilt beispielsweise für die Wirksamkeit einer Antibiotikatherapie bei COPD-Exazerbation. Ein Therapieerfolg kann sich nur einstellen, wenn die Exazerbation durch eine bakterielle Infektion ausgelöst wird, während das Medikament bei Exazerbationen anderer Ursache unwirksam ist. Bakterielle Exazerbationen kommen jedoch nur bei etwa 30\% der Ereignisse vor. In der ECLIPSE-Studie gab es Hinweise für einen „Exazerbierer“-Phänotyp: es handelte sich um Patienten mit konsistent häufigeren Exazerbationen im Verlauf der Erkrankung, die sich gleichzeitig durch stärkere 
Dyspnoe auszeichneten [1,2]. Die Verallgemeinerung von Studienergebnissen kann zu Fehlinterpretationen führen.

\section{Komplexität der Erkrankung und Studienendpunkte}

Seit mehreren Jahren begreift man die COPD als komplexe, multidimensionale Erkrankung. Viele unterschiedliche pulmonale und extrapulmonale Faktoren spielen zusammen, führen zur Krankheitsprogression und im Endstadium zur Invalidität. Es bleibt vor diesem Hintergrund eine Herausforderung, geeignete klinische Endpunkte zu finden.

Ein robuster, aber spät eintretender und unerwünschter Endpunkt ist das Versterben der Patienten. Häufig werden stattdessen Surrogatmarker verwendet, die die Wirkung einer Intervention auf den klinischen Endpunkt anzeigen sollen (z.B. FEV1, BODE-Index). Sie stehen nur in einer bedingten stochastischen Abhängigkeit zu den klinischen Endpunkten. Surrogatmarker sind zwar einfacher und früher messbar als das eigentliche Therapieziel. Ihre Verwendung bringt jedoch Probleme mit sich. Typischerweise wird in einer kleinen Studie zunächst nachgewiesen, dass die Intervention zu der gewünschten Änderung des Surrogatmarkers führt. Zur Prüfung der Hypothese wird dann eine große Interventionsstudie durchgeführt. Hier kann es dann zu unangenehmen Überraschungen kommen, wie bei der Prävention des Lungenkarzinoms mit Beta-Carotin und Alpha-Tocopherol. Epidemiologische Arbeiten konnten hier zeigen, dass Patienten mit Lungenkarzinom erniedrigte Vitaminspiegel hatten. In einer kleinen Studie wies man zunächst eine Normalisierung der Serumspiegel nach oraler Vitaminzufuhr nach. Außerdem wies die Placebogruppe eine höhere Inzidenz an Lungen-Karzinomen auf. Eine darauffolgende große Langzeitstudie zeigte dann zwar, dass sich bei Hochrisiko-Patienten durch Beta-Carotingabe der Vitaminspiegel normalisierte [3,4]. Jedoch fand man in der Verumgruppe $28 \%$ mehr Lungenkarzinom-Fälle als in der Placebo-Gruppe. Der klinische Endpunkt folgte also nicht dem Ergebnis des Surrogatmarkers.

Selbst die Letalität als robuster Endpunkt ist nicht ohne Probleme. So zeigte die TORCH-Studie [5], dass nur $1 / 3$ der Todesursachen direkt auf die Atemwegserkrankung zurückzuführen waren [6]. Die Mehrzahl der Patienten verstarb an kardiovaskulären (27\%) und onkologischen (21\%) Erkrankungen.

\section{Ausschlusskriterien und wirkliches Leben}

Ergebnisse klinischer Studien sollen möglichst für die gesamte Population der Erkrankten gelten. Um Behandlungseffekte optimal nachweisen zu können, werden für die Studienteilnahme nicht nur Einschluss-, sondern auch Ausschlusskriterien definiert. Flussdiagramme in den Publikationen stellen dar, wie viele der infrage kommenden Patienten in die Studie aufgenommen und am Ende ausgewertet wurden. Bei der VENT-Studie zu endobronchialen Ventilen bei COPD wurden 977 Patienten auf ihre Eignung zur Studienteilnahme überprüft, 321 wurden randomisiert und von nur 254 Personen lagen am Ende FEV1-Ergebnisse vor [7]. Es stellt sich die Frage, ob dieses kleine Kollektiv repräsentativ für die gesamte Patientengruppe ist. Diesem Thema der externen Validität gingen neuseeländische Autoren nach. Sie überprüften die 17 wichtigsten Studien, die der GINA-Leitlinie zugrunde liegen, und fassten die Ein- und Ausschlusskriterien dieser Studien zusammen. Bei einer postalischen Befragung von Patienten mit gesichertem Asthma zeigte sich, dass nur 4\% der Asthmatiker die Studienkriterien erfüllt hätten. Der Selektionsprozess stellt daher die Generalisierbarkeit der Ergebnisse infrage. Das Dilemma besteht darin, dass eine Aussage zum „wirk- lichen Leben“ gesucht wird, was bedeuten würde, möglichst wenige Ausschlusskriterien zu definieren. Klinische Prüfungen sind jedoch daraufhin angelegt, Hypothesen zu prüfen, und dies gelingt mit stark selektierten Patientengruppen leichter.

\section{Wichtige Studien zur COPD in 2010}

$\nabla$

\section{Referent: H. Teschler}

\section{COPD und Herz}

Kardiologen beachten zunehmend die respiratorische Komorbidität und Lungenfunktionsdaten als Risikofaktoren für kardiale Mortalität. Eine Auswertung des CREDO-Registers aus Kyoto, Japan, ergab unter den knapp 10000 analysierten Patienten mit koronarer Herzkrankheit eine Häufigkeit der COPD von 2,4\% [8]. Im Vergleich zu Patienten ohne COPD hatten Lungenkranke eine signifikant höhere Gesamtmortalität (Hazard Ratio 1,38), ein größeres Risiko für einen Herztod (HR 1,48) sowie eine höhere kardiovaskuläre Letalität (HR 1,28). Die COPD war demnach bei Patienten mit koronarer Herzkrankheit ein unabhängiger Risikofaktor für erhöhte Sterblichkeit.

Der Einfluss des Lungenemphysems auf die linksventrikuläre Füllung wurde von einer US-amerikanischen Arbeitsgruppe untersucht [9]. Bei 2816 Personen ohne bekannte kardiovaskuläre Erkrankung und mit einer mittleren FEV1 von 96\% des Solls wurden als spezielle Diagnostik Cardio-MRTs und Cardio-CTs durchgeführt. Das Ausmaß des Emphysems wurde mithilfe der Densitometrie bestimmt. Je ausgeprägter die emphysematösen Veränderungen oder die bronchiale Obstruktion waren, desto geringer war das linksventrikuläre enddiastolische Volumen. Offensichtlich wirken sich Veränderungen der Lunge ungünstig auf die Struktur und die Funktion des linken Ventrikels aus; Obstruktion und Emphysem reduzieren die linksventrikuläre Leistung. In einer Studie aus Großhansdorf wurde eine Korrelation zwischen Überblähung (ausgedrückt als Quotient IC/TLC) und dem enddiastolischen Durchmesser des linken Ventrikels beobachtet [10]. Ein Lungenemphysem kann also auch die Größe des Herzens beeinflussen.

Die Perfusion der Koronararterien bei Patienten mit und ohne COPD war Gegenstand einer weiteren Studie [11]. Dabei wiesen die COPD-Patienten signifikant höhere Entzündungsparameter (Fibrinogen und hsCRP) auf als die Kontrollgruppe. Der Blutfluss in den Herzkranzgefäßen war bei COPD deutlich reduziert und die mikrovaskuläre Myokardperfusion gestört, trotz normaler Koronarangiogramme.

Die COPD als Komorbidität wird von den Kardiologen also zunehmend wahrgenommen. Warum Obstruktion und Emphysem einen ungünstigen Einfluss haben, ist bisher noch nicht genau geklärt. Die systemische Inflammation der COPD könnte dabei eine Rolle spielen.

\section{COPD-Phänotypen}

In der ECLIPSE-Studie wurden Exazerbationen longitudinal über drei Jahre dokumentiert, und zwar bei mehr als 2000 COPD-Patienten mit den GOLD-Stadien II bis IV [2]. Die Definition einer Exazerbation war dabei äußerst streng. Je höher der Schweregrad der COPD, desto häufiger traten Exazerbationen auf und desto häufiger mussten die Patienten stationär behandelt werden. Ein entscheidender Prädiktor für das Auftreten von Exazerbationen war mindestens eine Exazerbation im Jahr vor Beginn der Auswertung. Wer im ersten Studienjahr eine Exazerbation durch- 
machte, hatte in den beiden Folgejahren ein höheres Risiko für weitere Exazerbationen. Diese Ergebnisse deuten auf phänotypische Unterschiede der analysierten Patienten hin. Ein zusätzlicher Risikofaktor war die Anamnese einer Reflux-Ösophagitis. Die therapeutische Konsequenz daraus ist jedoch noch unklar. Verschiedene COPD-Phänotypen wurden auch mithilfe von Cluster-Analysen identifiziert [12]. Die untersuchten 322 Patienten waren überwiegend in den GOLD Stadien II und III. Unter Berücksichtigung mehrerer Variablen beschrieb man schließlich vier phänotypische Kategorien: jung und schwer krank, jung und mittelgradig krank, alt und leicht krank sowie alt und schwer krank. Die Mortalität dieser vier Gruppen unterschied sich deutlich. Differenzierende Marker für den COPD-Phänotyp wurden in einem aktuellen Übersichtsartikel zusammengefasst [13].

\section{Neue Daten zu Tiotropium}

In einer neuen Auswertung der UPLIFT-Studie wurde der Langzeitverlauf der FEV1 mit und ohne Tiotropium differenziert nach dem Raucherstatus der Patienten dargestellt [14]. Das Ansprechen auf das Medikament war bei rauchenden und nicht mehr rauchenden Studienteilnehmern ähnlich. Allerdings wurde das Rauchverhalten nicht objektiviert und es handelt sich hier um eine post hoc Analyse. Inwiefern sich Tiotropium als erste Erhaltungstherapie bei COPD eignet, wurde in einer weiteren sekundären Analyse der UPLIFT-Daten ermittelt [15]. Von den 810 Patienten, die bei Studienbeginn noch keine Erhaltungstherapie erhalten hatten, wurden 403 in die Tiotropium- und 407 in die Placebo-Gruppe randomisiert. Auch bei dieser Analyse verbesserte Tiotropium die FEV1 signifikant stärker (um $134 \mathrm{ml}$ ) als Placebo. Zusätzlich wurde eine stärkere Verbesserung der gesundheitsbezogenen Lebensqualität nachgewiesen. Demnach eignet sich Tiotropium als erste Erhaltungstherapie bei COPD.

Patienten mit Asthma, die unter $2 \times 80 \mu \mathrm{g}$ BDP nicht ausreichend kontrolliert waren, erhielten in einer doppelblinden klinischen Prüfung entweder die doppelte Dosis von BDP $(2 \times 160 \mu \mathrm{g})$ oder eine Kombinationstherapie aus BDP $(2 \times 80 \mu \mathrm{g})$ plus Salmeterol $(2 \times 50 \mu \mathrm{g})$ oder BDP $(2 \times 80 \mu \mathrm{g})$ plus Tiotropium $(1 \times 18 \mu \mathrm{g})$ [16]. Nach 14-wöchiger Behandlung waren die morgendlichen Peakflow-Werte am Morgen in beiden Kombinationstherapie-Gruppen deutlich besser als unter doppelt so hoch dosiertem BDP. Auch der prozentuale Anteil der Tage mit guter Asthmakontrolle stieg stärker an. Bei diesen Asthmatikern waren die klinisch relevanten Therapieeffekte von Tiotropium und Salmeterol äquivalent.

\section{Nichtinvasive Beatmung}

In einer prospektiven Studie wurden 603 COPD-Patienten, die eine Langzeit-Sauerstofftherapie erhielten, polysomnografiert [17]. Man fand bei 95 Patienten eine obstruktive Schlafapnoe und bot ihnen eine CPAP-Therapie an. Die 61 Patienten, die die nichtinvasive Beatmung akzeptierten, hatten eine sehr viel höhere 5-Jahres-Überlebensrate als die Gruppe ohne CPAP (71\% vs. $26 \%$ ). Eine andere Arbeit berichtete über den Langzeitverlauf von Patienten mit Overlap-Syndrom, die mit CPAP $(n=228)$ oder ohne CPAP ( $n=213$ ) behandelt wurden [18]. Innerhalb von 12 Jahren war das exazerbationsfreie Überleben der Overlap-Patienten mit CPAP praktisch identisch mit der von COPD-Patienten ohne Overlap-Syndrom, während die nicht mit CPAP behandelten Patienten deutlich schlechter abschnitten. Dieselben Unterschiede ergaben sich hinsichtlich der Überlebensrate der Patienten. Das Overlap-Syndrom war mit gesteigerter Morbidität und Mortalität assoziiert, wobei eine CPAP-Therapie die Prognose verbesserte. Dies galt auch für Patienten unter Langzeitsauerstofftherapie.

Eine mögliche Erklärung für die Wirksamkeit von CPAP könnte darin liegen, dass die nächtliche Überdruck-Therapie die Refluxrate deutlich reduziert. Mini-Aspirationen könnten seltener werden. Auch der Transit von Keimen des Mundes in die tiefen Atemwege hinein während des Schlafs könnte sich durch CPAP verringern. Und schließlich ist denkbar, dass CPAP zu einer Verbesserung der Pumpfunktion des Herzens und zu einer Reduktion von Herzrhythmusstörungen beiträgt.

\section{Definition der COPD in klinischen Studien $\nabla$}

\section{Referent: A. Gillissen}

Grundsätzlich gilt für jede klinische Prüfung, dass das Studiendesign, die Patientenselektion, die Auswahl des Studienendpunktes und die Begleitmedikation das Ergebnis stark beeinflussen. Vergleicht man die COPD-Definition nach GOLD mit den Einschlusskriterien für Patienten in den großen COPD-Studien mit mehreren Tausend Teilnehmern, so ergibt sich insgesamt keine gute Übereinstimmung.

\section{UPLIFT}

Zur Definition der COPD wurden in der UPLIFT-Studie die Patientenunterlagen der letzten fünf Jahre herangezogen [19]. So erreichte man eine klare Beschreibung des Patientenkollektivs. Die Patienten mussten mindestens 40 Jahre alt sein und eine Raucheranamnese von mindestens 10 Packungsjahren aufweisen, ihre postbronchodilatatorische FEV1 musste $\leq 70 \%$ des Solls betragen und der Quotient FEV1/FVC geringer als 70\% sein. Zu den Ausschlusskriterien gehörte ein Asthma bronchiale, wobei hier lediglich die anamnestische Angabe des Patienten herangezogen wurde. Es gab zwei gleichwertige primäre Endpunkte, nämlich den mittleren jährlichen Abfall der FEV1 sowohl vor als auch nach Bronchodilatation. Zu den sekundären Endpunkten gehörten neben weiteren Lungenfunktionskriterien die krankheitsbezogene Lebensqualität, die Rate der COPD-Exazerbationen sowie die Gesamtmortalität. Bereits im Methodenteil der Publikation wurde angekündigt, dass bestimmte Subgruppenanalysen geplant waren.

\section{TORCH}

In der TORCH-Studie war die Definition der COPD weniger strikt $[5,20]$. Es reichte eine fundierte Anamnese („established history“) der COPD, sodass nicht auszuschließen ist, dass Patienten mit einem jahrelangen Asthma eingeschlossen wurden. Jedoch wurde hier die FEV1-Reversibilität gemessen, die nach Salbutamol maximal 10\% betragen durfte. Der primäre Endpunkt dieser Studie war die Zeit bis zum Tod, wobei alle Todesursachen berücksichtigt wurden. In zwei während der laufenden Studie durchgeführten Interims-Analysen wurden die statistischen Signifikanzkriterien adaptiert und damit verschärft.

\section{ECLIPSE}

Bei der ECLIPSE-Studie war das Höchstalter der Studienteilnahme auf 75 Jahre begrenzt [21]. Die Patienten waren weniger schwer krank als in den beiden anderen Studien und die Definition der COPD war weniger strikt. Zu den Ausschlusskriterien gehörten diverse Begleiterkrankungen und Behandlungen, beispielsweise Zustand nach Lungenchirurgie oder die Einnahme oraler Steroide. Da es sich primär um eine Beobachtungsstudie 
handelte, wurde kein primärer Endpunkt definiert. Zentraler Parameter war die Lungenphysiologie, ergänzt durch volumetrische CT-Scans mit niedriger Strahlendosis. Außerdem wurden klinische Verlaufsparameter dokumentiert, Biomarker im Blut und anderen Körperflüssigkeiten gemessen, und die Patienten beantworteten Fragebögen zur Lebensqualität, Dyspnoe und Depression.

\section{BRONCUS}

In diese N-Acetylcystein-Studie wurden rauchende COPD-Patienten eingeschlossen. Außerdem mussten die Studienteilnehmer über zwei Jahre mindestens zwei COPD-Exazerbationen pro Jahr durchgemacht haben [22]. Die FEV1-Reversibilität musste weniger als $12 \%$ und die postbronchodilatatorische FEV1/FVC-Ratio weniger als $88 \%$ betragen. Zu den Ausschlusskriterien gehörte nicht nur ein anamnestisches Asthma, sondern auch eine allergische Rhinitis mit oder ohne Ekzem. Primäre Endpunkte waren der Abfall der FEV1 pro Jahr sowie die Rate an COPD-Exazerbationen.

\section{Roflumilast}

Für die beiden aktuellen Studien zu Roflumilast wurde die COPD ähnlich wie bei TORCH definiert, und es mussten bei den Patienten zusätzlich die Kriterien einer chronischen Bronchitis erfüllt sein. Die Exazerbationsrate der Patienten wurde nur anamnestisch erhoben $[23,24]$. Auch hier gehörte das Asthma zu den Ausschlusskriterien. Ferner durften die Patienten nicht ohne Husten und Auswurf sein. Bei den primären Endpunkten Abfall der FEV1 und Exazerbationsrate wurde auch die Schwere der Exazerbation mitberücksichtigt, indem Krankenhausaufnahmen oder Mortalität von leichteren Exazerbationen unterschieden wurden.

\section{Ziele der COPD-Therapie nach GOLD}

Nach den aktuellen GOLD-Empfehlungen soll die Therapie die Symptomatik und die Progression der Erkrankung reduzieren. Die Patienten sollen besser belastbar sein, eine bessere Lebensqualität haben und länger leben. Vermieden werden sollen Komplikationen einschließlich COPD-Exazerbationen und Nebenwirkungen der Medikamente.

Nicht jedes dieser Therapieziele kann in Studien erfasst werden. Zum Thema Studienendpunkte bei COPD erschienen diverse Übersichtsarbeiten, die sich mit dieser Problematik beschäftigen $[25,26]$. Es werden geeignete Surrogat-Parameter benötigt, die verlässlich die Komplexität der Erkrankung anzeigen. Dies ist eines der wesentlichen Ziele der ECLIPSE-Studie. Von den Behörden (und IQWIG) wird z.B. die Lungenfunktion und hier insbesondere die FEV1 nur als Surrogat-Parameter, nicht aber als patientenrelevanter Endparameter angesehen, womit ein großer Teil der vorliegenden COPD-Studien für die Wirksamkeit von Pharmaka nicht oder nur partiell anerkannt werden. Als patientenrelevant gelten lediglich die Verlängerung der Überlebenszeit, die Verbesserung der Lebensqualität und die Verringerung des Dyspnoeempfindens.

\section{Lungenvolumina als Zielparameter}

$\nabla$

\section{Referent: H. Worth}

Die Messung unterschiedlicher Lungenvolumina ist sinnvoll zur Diagnosestellung und Differentialdiagnose der COPD. Die Ergebnisse erlauben Aussagen zur Beeinträchtigung des Patienten und zur Abschätzung seiner Prognose. Nach der deutschen COPDLeitlinie [27] werden Lungenvolumina zur Einteilung des Schweregrads der Erkrankung herangezogen, gehören also zur Basisdiagnostik.

Der Quotient FEV1/FVC, der bei COPD unter 70\% liegt, markiert zwar die Obstruktion, er eignet sich jedoch nicht zur Quantifizierung des Emphysems. Zudem resultiert bei älteren Patienten ein erheblicher Anteil falsch positiver Ergebnisse. Daher wurde angeregt, anstelle eines festen Quotienten die untere Grenze der altersabhängigen Normwerte heranzuziehen [28]. Andere Autoren schlugen den Quotienten FEV1/FEV6 vor, um eine bessere Reproduzierbarkeit zu erreichen und den Altersgang zu berücksichtigen $[29,30]$.

Auch die Aussagekraft des Bronchospasmolysetests ist limitiert, denn ein Anstieg der FEV1 nach Bronchodilatation um mehr als 12 bis $15 \%$ bzw. $200 \mathrm{ml}$ erlaubt weder die Separation zwischen Asthma und COPD noch eine Aussage zum voraussichtlichen Ansprechen des Patienten auf eine Therapie mit Bronchodilatatoren.

\section{FEV1}

In zahlreichen klinischen Prüfungen wurde die Veränderung der FEV1 als Studienendpunkt verwendet. In Kurzzeitstudien wird ein Anstieg um mindestens 20\% und bei Studien über mehr als ein Jahr eine Verbesserung um 15\% als relevant angesehen. Nachgewiesen wurde eine Assoziation der präbronchodilatatorischen FEV1 mit der Sterblichkeit.

In den letzten Jahren wurden diverse Limitationen der FEV1 publiziert. So korreliert die postbronchodilatatorische FEV1 schlecht mit der Belastbarkeit der Patienten, der Dyspnoe und der krankheitsspezifischen Lebensqualität [31]. Das subjektive Empfinden der Atemnot kommt besser zur Darstellung, wenn das forcierte inspiratorische Volumen in einer Sekunde (FIV1) gemessen wird [32]. Um die Besserung der Lungenfunktion nach einer Exazerbation der COPD zu erfassen, wurde die FEV1 mit anderen Kenngrößen verglichen [33]. Die Veränderungen zwischen Entlassungstag und Aufnahmetag waren für die FEV1 geringer als für die inspiratorische Kapazität. Auch 42 Tage nach Krankenhausaufnahme waren beide Werte signifikant höher als vor Beginn der Behandlung. Bei Patienten mit schwerer COPD wurde die Füllung des linken Ventrikels gemessen und mit den FEV1Werten verglichen [9]. Dabei ergab sich eine schlechte Korrelation, während der Bezug zum Ausmaß des Emphysems, das mithilfe von CT-Aufnahmen ermittelt wurde, besser war.

Bearbeitet wurde auch die Frage, wie groß für die FEV1 der minimale klinisch relevante Unterschied ist [31]. Leitlinien nennen $200 \mathrm{ml}$ oder $12 \%$, während Patienten subjektiv bereits Differenzen von $112 \mathrm{ml}$ oder $4 \%$ als Unterschied empfinden. Wenn neue Festlegungen zum relevanten Unterschied erfolgen sollen, müsste die Schwere der Erkrankung berücksichtigt werden. Schließlich wurde in den letzten Jahren nachgewiesen, dass die FEV1 allein die Überlebenswahrscheinlichkeit der Patienten schlechter vorhersagt als kombinierte Indizes wie der BODE-Index [34]. 


\section{Andere Lungenvolumina}

Lungenvolumina wie funktionelle Residualkapazität oder Residualvolumen sind schwerer zu messen als die FEV1. Sie erfordern eine Bodyplethysmografie oder eine Spirometrie mit Inertgasanalyse.

Bei der COPD korrelieren FRC und inspiratorische Kapazität besser mit Belastbarkeit und Dyspnoe als die FEV1. Die inspiratorische Kapazität hat sich als unabhängiger Prädiktor der Sterblichkeit erwiesen. Im Verlauf von Exazerbationen reflektiert sie die Änderung der Überblähung. Außerdem kann sie als Indikator für die inspiratorische Atemmuskelkraft verwendet werden.

Eine dynamische Überblähung mit stark erhöhtem Residualvolumen ist bei einem Teil der COPD-Patienten ein erhebliches Problem [35]. Durch eine Behandlung mit Tiotropium nimmt das Residualvolumen ab, die FRC steigt und die Patienten haben eine bessere Ausdauerleistung [36]. Als Kenngröße der Atemmuskelfunktion kann auch die FIV1 herangezogen werden. Ein neunmonatiges ambulantes körperliches Training mit oder ohne zusätzliches Atemmuskeltraining führte zu einer signifikanten Verbesserung der FIV1 um 11\% gegenüber dem Ausgangswert (Worth, eigene unveröffentlichte Daten).

Der Quotient IC/TLC eignet sich auch als prognostischer Indikator. Eine Analyse bei knapp 700 COPD-Patienten ergab über 34 Monate eine signifikant schlechtere Prognose, wenn dieser Wert kleiner als 25\% war [37]. Bezüglich der Validität der Prädikation liegt der IC/TLC-Quotient zwischen FEV1 und BODE-Index.

Zusammengefasst haben die verschiedenen Lungenvolumina unterschiedliche Stärken und Schwächen hinsichtlich ihrer Eignung als Endpunkt in klinischen Studien. Die FEV1 als einfacher und gut messbarer Parameter ist am besten untersucht. Sie korreliert jedoch nicht mit Exazerbationen und Lebensqualität. Mit der FIV1 kann man die Dyspnoe recht gut erfassen, es gibt jedoch weniger zuverlässige Sollwerte als für die FEV1. Der Quotient FEV1/FVC ist anwendbar, sofern man sehr niedrige Sollwerte ansetzt. Die Messung der inspiratorischen Kapazität bietet sich an, um die dynamische Lungenüberblähung zu erfassen, es gibt jedoch noch keine zuverlässigen Sollwerte. Als Prognoseindikator eignet sich der Quotient IC/TLC gut. Residualvolumen und funktionelle Residualkapazität allein sind keine guten Endpunkte und sollten daher auf die TLC bezogen werden.

\section{Exazerbationen \\ $\nabla$}

\section{Referent: W. Randerath}

Exazerbationen sind bei COPD-Patienten die häufigste Ursache für Arztbesuche, stationäre Aufnahmen und Letalität [38]. Heutzutage besteht der Eindruck, dass die Patienten insgesamt stabiler sind als früher. Auch eine Analyse wichtiger COPD-Studien bestätigt diese Tendenz [39]: Seit 1970 nahmen die in Studien dokumentierten Exazerbationen von ursprünglich 2,3 pro Patient und Jahr [40] auf zuletzt nur noch 0,85 pro Patient und Jahr ab [19].

\section{Studienplanung}

Ursachen dafür liegen unter anderem bei den Einschlusskriterien. Die Definition der COPD hat sich im Verlauf von 30 Jahren geändert. So spielt es eine Rolle, ob Patienten mit Reversibilität in die Studie aufgenommen oder ob bestimmte Subtypen ausgeschlossen wurden. Im Unterschied zu früher basieren aktuelle Definitionen der Exazerbation nicht nur auf einer Zunahme der Symptome, sondern erfordern zusätzlich eine Änderung der Me- dikation. In der INSPIRE-Studie reduzierte diese Festlegung die Zahl der Exazerbationen von 3 auf 1,5 pro Jahr [41]. Hinsichtlich der Exazerbationsfrequenz spielt eine Rolle, ob man sich auf anamnestische Angaben des Patienten verlässt, oder ob es sich um dokumentierte Beobachtungen bei relativ rasch aufeinander folgenden Studienbesuchen handelt. Auch regelmäßige Telefonate mit Patienten oder die Nutzung von Tagebüchern wirken sich auf die Erfassung von Exazerbationen aus.

\section{Krankheitsverlauf}

Bei Patienten mit häufigen COPD-Exazerbationen war die Abnahme der FEV1 im Langzeitverlauf signifikant größer $[42,43]$. Auch die Lebensqualität wurde durch häufige Exazerbationen stärker eingeschränkt, zumindest bei Patienten mit einer FEV1 zwischen 35 und 50\% des Solls [44]. Exazerbationen, die während der Studie vom Patienten nicht selbst berichtet wurden, sondern lediglich aus der Studiendokumentation hervorgingen, wirkten sich ebenfalls ungünstig auf die krankheitsbezogene Lebensqualität aus [45].

Offenbar gibt es den Phänotyp des „häufig exazerbierenden Patienten“. Diese Betroffenen haben zahlreiche Exazerbationen in der Vorgeschichte, häufiger Symptome der Bronchitis oder der Obstruktion und in der Regel eine stärker eingeschränkte FEV1. Auch bei der Untersuchung von Biomarkern zeigte diese Subgruppe stärkere Auffälligkeiten [46]: das CRP im Serum war höher, das OPG (Osteoprotegrin) niedriger, und es bestanden Korrelationen zwischen STNFR-1 und OPG mit dem GOLD-Stadium. Zusätzlich wurde eine Kolonisation mit Krankheitserregern wie H. influenzae, S. pneumoniae, H. parainfluenzae, Branhamella und Pseudomonas species häufiger beobachtet [47]. In derselben Untersuchung war das IL-8 im induzierten Sputum umso höher, je größer die Bakteriendichte war.

\section{Antibiotikatherapie}

Es scheint naheliegend, die Bakterienlast in den Atemwegen mit einer kontinuierlichen oder intermittierenden Antibiotikatherapie zu reduzieren. In einer Studie mit Moxifloxacin über 5 Tage war nach 2 Wochen der Anteil bakterienbesiedelter Patienten mit 30\% signifikant geringer als in der Placebogruppe mit 75\% [48]. Allerdings fand man bei der Kontrolluntersuchung nach 8 Wochen kaum noch Unterschiede zwischen den beiden Studiengruppen. Das Konzept einer 5-tägigen Pulstherapie mit Moxifloxacin alle 8 Wochen ergab zwar eine Verbesserung der Lebensqualität unter Verum [49], jedoch bestanden über 48 Wochen keine Vorteile hinsichtlich Hospitalisationsrate oder Mortalität und die Nebenwirkungsrate unter Moxifloxacin war erhöht. Daraus ließ sich keine Empfehlung für eine antibiotische Pulstherapie ableiten.

\section{Inhalative Steroide}

Viel diskutiert wird der Einfluss inhalativer Steroide auf die Häufigkeit von Exazerbationen. Einige Studien zeigten gute Effekte, andere dagegen keine Wirkung. Eine kritische Re-Analyse der publizierten Studien fasste die methodischen Probleme zusammen [50]. Wenn Patienten mit inhalativen Steroiden vorbehandelt worden waren, hatte diese Subgruppe häufigere Exazerbationen als nicht vorbehandelte Patienten. Rechnet man diese ca. 50\% der Studienteilnehmer heraus, fand sich kein Unterschied der Exazerbationsrate mehr. In einigen Studien wurden die Patienten nach der ersten Exazerbation nicht weiter nachbeobachtet. Analysiert man dagegen die Zeit bis zur zweiten Exazerba- 
tion, ergaben sich keine Unterschiede zwischen der Therapie mit oder ohne inhalative Steroide [50].

Zur Häufigkeit von Pneumonien unter einer ICS-Therapie zeigte sich in einer Nach-Analyse der INSPIRE-Studie, dass mit einer steroidhaltigen Kombination (Salmeterol/Fluticason) behandelte Patienten häufiger an Pneumonien erkrankten als Patienten, die Tiotropium erhalten hatten [51]. Diskutiert wurde zudem der Einfluss von Rehabilitationsprogrammen [52], die Nachteile durch die Unterbrechung der Anticholinergika-Therapie in den Placebo-Gruppen [53] und fehlende Informationen zu Beginn und Ende neuer Therapien mit ICS oder langwirksamen Betasympathomimetika (LABAs) $[53,54]$.

\section{Bronchodilatatoren}

Die Analyse einer Subgruppe von UPLIFT-Patienten, die bei Randomisation keine Erhaltungstherapie der COPD angegeben hatten, ergab zwar eine signifikante Verbesserung der Lungenfunktion unter Tiotropium, jedoch keine Unterschiede hinsichtlich der Häufigkeit von Exazerbationen oder der Zeit bis zur ersten Exazerbation [15,53]. Allerdings war die Exazerbationsrate insgesamt niedrig und schränkte daher die statistische Power dieser Analysen ein.

Dass Hausärzte heutzutage über eine geringere Exazerbationsrate ihrer COPD-Patienten als früher berichten, kann mit der besseren Langzeit-Bronchodilatation zusammenhängen. Eine starke Überblähung der Lunge erfordert eine höhere Atemarbeit und erzeugt dadurch eine Dyspnoe. Bei besserer Lungenfunktion ist die Atemnot geringer. Letzten Endes ist es der Patient, der bestimmt, mit welchen Beschwerden er zum Arzt geht. Eine Abnahme der Dyspnoe und der übrigen Symptomatik könnte daher für die geringere Exazerbationsrate eine Rolle spielen.

Weniger Exazerbationen wurden auch für den Phosphodiesterase-Hemmer Roflumilast beschrieben [23,53]. Die Behandlung reduzierte die jährliche Exazerbationsrate pro Patient gegenüber Placebo um 17\%. Dieser Effekt dürfte auf die antiinflammatorische Wirkung der Substanz zurückzuführen sein, denn ein klinisch relevanter bronchodilatatorischer Effekt wurde unter Roflumilast nicht beobachtet.

Zusammenfassend gibt es derzeit zwei Wege, um die Häufigkeit von Exazerbationen medikamentös zu reduzieren. Die suffiziente Bronchodilatation verbessert die FEV1 und reduziert das Residualvolumen. Eine antiinflammatorische Therapie kann ebenfalls günstige Effekte auf die Exazerbationsrate haben.

\section{Körperliche Aktivität und Belastbarkeit}

\section{$\nabla$}

\section{Referent: H. Magnussen}

Bereits im Jahr 1994 publizierte die Arbeitsgruppe aus Großhansdorf eine Faktorenanalyse, nach der die Schweregrade der COPD durch die Gehstrecke, die Überblähung und die Atemwegsobstruktion charakterisiert werden können [55]. Der BODE-Index [34], der zusätzlich den Ernährungszustand ausgedrückt als BMI berücksichtigt, kann als Fortführung dieses Ansatzes betrachtet werden.

Zur Quantifizierung der Belastbarkeit einer Person stehen verschiedene Methoden zur Verfügung. Etabliert sind vor allem die Ergometrie und der 6-Minuten-Gehtest. In den letzten Jahren gewann die fortlaufende Erfassung der körperlichen Aktivität im Alltag an Bedeutung, die mit Hilfe eines Pedometers oder eines Accelerometers dokumentiert werden kann.

\section{Ergometrie}

Körperliche Belastbarkeit und Ausdauer können durch Bronchodilatatoren wie Tiotropium günstig beeinflusst werden. Eine sechswöchige Behandlung reduzierte das Residualvolumen und damit die Überblähung und steigerte die ergometrisch gemessene Ausdauer signifikant im Vergleich zu Placebo [36].

\section{6-Minuten-Gehtest}

Der 6-Minuten-Gehtest (6 MWT) erfasst die Antwort aller Organsysteme auf eine körperliche Belastung [56]. Er wird nicht nur in der Pneumologie eingesetzt, sondern auch in der Kardiologie und bei der Adipositas-Therapie. Seine Standardisierung verlangt geeignete räumliche Verhältnisse mit einem 30 m langen Korridor und beinhaltet genaue Instruktionen, wie der Patient angeleitet und ermuntert werden muss. Das Erfassen der Dyspnoe erfolgt mithilfe der Borg-Skala. Der Normwert für den 6 MWT liegt im Bereich von $600 \mathrm{~m}$, wobei einige Autoren für 60- bis 65-jährige gesunde Personen einen Mittelwert von 527 [57] und andere einen von $631 \mathrm{~m}$ [58] angaben.

Die Streuung bei gesunden Personen ist erheblich. Bei Patienten mit COPD war die Gehstrecke in Abhängigkeit von der FEV1 deutlich reduziert und lag bei durchschnittlich 366 bzw. $440 \mathrm{~m}$ $[59,60]$. Die ECLIPSE-Studie ergab eine verkürzte Gehstrecke unterhalb 350 m bereits bei 31\% der Patienten im GOLD Stadium II; im Stadium III waren es $47 \%$ der Patienten [61].

Die Dyspnoe-Empfindung und damit die Gehstrecke wird zusätzlich durch Umgebungsfaktoren und durch psychologische Faktoren beeinflusst. Hörten Patienten während des Belastungstests angenehme Musik, gaben sie signifikant weniger Dyspnoe an und empfanden den Test als weniger unangenehm [62].

Therapeutische Maßnahmen wirken sich positiv auf die Gehstrecke aus. So wurde durch Rehabilitation eine Verbesserung um 30 bis 84 m erreicht $[63,64]$ oder durch Lungenvolumenreduktionschirurgie eine Steigerung um 32 bis 96 m [65]. Durch Bronchodilatation konnte die Gehstrecke um 27 m [66] und durch Sauerstoffgabe während der Belastung um 95 m [67] erhöht werden. Als therapeutisch relevante Verbesserung der Strecke wurde ein Anstieg um $35 \mathrm{~m}$ angesehen [68].

Das Ergebnis des 6-Minuten-Gehtests diente als Ausgangspunkt für ein Rehabilitationsprogramm nach akuter Exazerbation einer COPD [69]. Dabei sollten die Patienten fünfmal am Tag eine Strecke gehen, die 75\% ihrer 6-Minuten-Gehstrecke entsprach. Außerdem erhielten sie eine 60-minütige Schulung pro Woche sowie 30 Minuten Physiotherapie pro Tag. Zehn Tage nach Beginn des Trainings hatten FEV1 und 6-Minuten-Gehstrecke signifikant zugenommen. Durch Fortführung eines vergleichbaren Programms zuhause über sechs Monate ließen sich die Verbesserungen aufrechterhalten, im Unterschied zur Entwicklung in der Kontrollgruppe [70]. Eine andere Studie bei hospitalisierten Patienten mit Exazerbation ergab günstige Auswirkungen eines Widerstandstrainings auf die Kraft des Quadriceps, auf die systemische Entzündung und auf Veränderungen in der Muskelbiopsie [71]. Der Effekt war umso deutlicher, je stärker beeinträchtigt die Patienten waren.

\section{Tägliche körperliche Aktivität}

Körperliche Aktivität wird verstanden als jegliche durch Muskelaktivität ausgelöste körperliche Bewegung, die in einem Energieverbrauch oberhalb des Ruhezustands resultiert [72]. Zur Messung der Alltagsaktivität eignen sich Schrittzähler (Pedometer), Beschleunigungssensoren (Accelerometer) sowie das Sense- 
Wear ${ }^{\circledR}$-Armband, das zusätzlich physiologische Sensoren zur Erfassung von Hautwiderstand und Körpertemperatur enthält.

Eine relativ einfache Methode ist die Messung der Schritte, die eine Person pro Tag zurückgelegt. Bei COPD wird damit jedoch nicht der erhöhte Energiebedarf erfasst, der zum Beispiel durch eine verstärkte nächtliche Atemarbeit erklärt werden kann. Diese Zusammenhänge können als Physical Activity Level (PAL) ausgedrückt werden, der durch den totalen täglichen Energieverbrauch geteilt durch den Energieverbrauch während des Nachtschlafs definiert ist. Gesunde körperlich aktive Menschen haben einen PAL von über 1,7, während inaktive Personen ein PAL unter 1,4 haben.

Die körperliche Aktivität ist signifikant mit der Mortalität (alle Ursachen) assoziiert: das Mortalitätsrisiko war bei moderater Aktivität rund $20 \%$ geringer als bei inaktiven Personen [73]. Bei COPD war bereits im Stadium GOLD I die tägliche Schrittzahl gegenüber einer Kontrollgruppe leicht erniedrigt [74]. Offenbar schränkt bereits die frühe COPD den Lebensstil ein. Es wäre wichtig, mehr für diese gering Betroffenen zu tun.

Das SenseWear®-Armband erfasst den Energieverbrauch sehr zuverlässig. Dies zeigte der Vergleich mit der Goldstandardmethode, der indirekten Kalorimetrie [75]. Neue unveröffentlichte Ergebnisse, die mit dem SenseWear ${ }^{\circledR}$-Armband erhoben wurden, legen nahe, dass körperlich aktive COPD-Patienten deutlich länger leben als inaktive. Wer im Alltag beweglich ist, trainiert sich fortlaufend selbst. Zukünftig lassen sich auf relativ einfache Weise Gruppen von Patienten identifizieren, die sich mehr oder weniger bewegen.

\section{Biomarker bei der COPD \\ $\nabla$}

\section{Referent: R. Bals}

Biomarker spielen in der aktuellen Forschung eine wichtige Rolle. Nach einer Definition des NIH handelt es sich um einen objektiv gemessenen Indikator, der einen normalen biologischen bzw. einen Krankheitsprozess oder die pharmakologische Antwort auf eine therapeutische Intervention anzeigt.

Für die COPD wurden bisher noch keine Biomarker etabliert. Wünschenswert wäre beispielsweise ein Biomarker für die Therapiesteuerung, ähnlich wie das HbA1C beim Diabetes mellitus, oder ein diagnostischer Biomarker wie das Troponin, das ein akutes Koronarsyndrom anzeigt. Eine Untergruppe der Biomarker sind die Surrogatmarker, die einen klinischen Endpunkt ersetzen können, weil sie als Indikator für das klinische Ansprechen von Patienten gelten.

Die Diagnose der COPD wird klinisch gestellt. Funktionsanalysen und Bildgebung unterstützen bei der Beurteilung der aktuellen Problematik und der Einstufung des Schweregrades. Biomarker könnten bei der COPD unterschiedliche Funktionen erfüllen: Sie können die genetische Prädisposition für die Erkrankung anzeigen, sie können als Prognose-Marker dienen und sie können in klinischen Studien eingesetzt werden.

\section{Genetische Prädisposition für COPD}

Für die COPD wurden diverse Kandidatengene wissenschaftlich untersucht [76]. Als validiert gilt das SERPINA1-Gen für den Alpha-1-Antitrypsin (AAT)-Mangel. Das AAT als Biomarker ist sehr einfach zu messen. Die moderne Testung auf Filterpapier ermöglicht die nephelometrische Bestimmung des AAT-Gehalts, die PCR auf S- oder Z-Allele sowie die isoelektrische Fokussierung.
Geringe Serumkonzentrationen von AAT führten bei Betroffenen zur rascheren Verschlechterung der Lungenfunktion [77].

Als weiterer Biomarker bei chronisch obstruktiver Lungenerkrankung gilt die Matrix-Metalloproteinase MMP12 [78]. In einer Analyse von sieben Kohorten mit zusammen mehr als 8000 Personen ließ sich zeigen, dass eine bestimmte Mutation mit einer 35-prozentigen Abnahme des COPD-Erkrankungsrisikos assoziiert war.

\section{Prognose der COPD}

In einer Übersichtsarbeit wurden prognostische Marker für den Langzeitverlauf der COPD zusammengestellt [79]. So fand man bei erhöhten Fibrinogen-Konzentrationen eine höhere Hospitalisierungsrate [80]. Das CRP als klassischer Parameter für systemische Entzündungsprozesse war bei Rauchern mit COPD erhöht [81]. In der Kopenhagen-City-Heart-Studie wurde untersucht, ob das CRP kausal an der COPD-Progression beteiligt ist [82]. Es fanden sich Korrelationen zwischen CRP und FEV1 sowie zwischen CRP-Genotyp und CRP-Konzentrationen. Die weitere Analyse ergab jedoch, dass das Auftreten einer COPD unabhängig vom CRP-Genotyp war, dass also keine kausale Beziehung zur Erkrankung bestehen dürfte.

\section{Klinische Endpunkte}

In den letzten Jahren wurde die Bedeutung des Surfactant Protein D für die COPD untersucht. Es handelt sich um ein Protein aus den Strukturzellen der Lunge, das antiinflammatorische und antioxidative Wirkungen ausübt und eine der Komponenten der angeborenen Immunität ist. In einer kleinen Studie an 23 COPDPatienten fanden die Autoren nur eine mäßige Korrelation zwischen der FEV1 und den Konzentrationen von Surfactant Protein D im Serum [83].

Das Clara cell secretory protein-16 (CC-16) wird in den Clara-Zellen der Lunge produziert. Seine Bestimmung bei Patienten in verschiedenen GOLD-Stadien ergab nur minimale Unterschiede zwischen den Gruppen, die weder klinisch noch für Studien relevant sind [84].

Bildgebende Verfahren wie das CT der Lunge wurden ebenfalls auf ihre Eignung als Biomarker hin untersucht. So wurden in einer kleineren Studie die regionale Perfusion und der pulmonale Blutfluss gemessen [85]. Bei Rauchern mit beginnendem Emphysem war die Perfusion heterogener als bei Rauchern mit normalem Lungen-CT. Mit der Computertomografie ließen sich zwar charakteristische Muster beschreiben, die Methode eignet sich aus Sicht der FDA jedoch nicht als Biomarker für die COPD.

Eine Übersicht über biologische, physiologische und symptomatische Marker der COPD zeigt die bunte Vielfalt der Forschungsaktivitäten [86]. Die Validierung eines neuen Markers, der sich auch für Studien eignet, ist nicht trivial. Vor allem muss nachgewiesen werden, dass der Marker auf die Intervention reagiert und damit als Substitut für das klinische Ansprechen gelten kann. Es bleibt zu hoffen, dass das Kompetenznetz COPD/Asthma mit seiner nationalen COPD-Kohortenstudie COSYCONET dazu einen Beitrag leisten wird. 


\section{BODE-Index als kombinierter Endpunkt}

\section{$\nabla$}

\section{Referent: G. Schultze-Werninghaus}

Die Publikation zum BODE-Index von Celli u. Mitarb. datiert aus dem Jahr 2004 [34]. Der Index setzt sich aus vier Kriterien zusammen: Body Mass Index, Atemwegsobstruktion, Dyspnoe und körperliche Belastbarkeit. Die Messparameter sind FEV1 (in \% des Solls), 6-Minuten-Gehstrecke (in m) und die Dyspnoe-Skala nach dem MMRC-Fragebogen. Der Ernährungszustand wird in nur zwei Kategorien unterteilt, nämlich BMI $>21$ oder $\leq 21 \mathrm{~kg} / \mathrm{m}^{2}$, während es für die anderen Parameter jeweils vier Kategorien ( 0 bis 3 Punkte) gibt. Die beste Punktzahl beträgt 0 und die schlechtestmögliche 11. Die Autoren konnten zeigen, dass der BODE-Index gut mit der Überlebenswahrscheinlichkeit korreliert.

\section{Kritische Anmerkungen}

Schon kurz nach der Publikation gab es kritische Äußerungen. So wurde bemängelt, dass eine Kategorie für übergewichtige Personen fehlt. Andere Kritikpunkte waren, dass Patienten mit Herzinsuffizienz oder anderen Erkrankungen mit erhöhter Letalität in der Analyse von Celli ausgeschlossen waren und dass wesentliche prädiktive Faktoren für die Mortalität nicht berücksichtigt wurden, beispielsweise Raucherstatus oder Exazerbationshäufigkeit [87]. Die Kaplan-Meier Überlebenskurven der Originalarbeit zeigten außerdem über die vierjährige Beobachtungszeit einen großen Verlust an Patienten von ursprünglich 624 auf am Ende nur noch 48 Patienten. Selbst von den 169 Patienten der ,gesündesten“ Quartile 1 waren nach vier Jahren nur noch 19 Personen in der Kohorte verblieben.

\section{Modifikationen}

Zwischen 2005 und 2010 erschienen sechs Publikationen mit Vorschlägen zur Modifikation des BODE-Index. So wurde beispielsweise die Exazerbationshäufigkeit ergänzt [88] oder der 6Minuten-Gehtest durch die Sauerstoffaufnahme ersetzt [89]. Auch Puhan et al. stellten den BODE-Index infrage und wiesen nach, dass die Kriterien Alter, Dyspnoe und Obstruktion (ADO) ebenso gute prognostische Marker sind [90]. Im ambulanten Bereich wurde als Alternative der HADO-Index untersucht, der aus den Komponenten Gesundheit ( $\mathrm{H}$, health), Aktivität, Dyspnoe und Obstruktion zusammengesetzt ist [91]. Für die respiratorische wie für die Gesamtmortalität war der HADO-Score ein vergleichbar guter Prädiktor wie der BODE-Index.

\section{Prognostische Aussagekraft}

Die Eignung des BODE-Index als Prädiktor wurde in neun verschiedenen Arbeiten untersucht. Im Vergleich zur FEV1 erwies sich der Index als besserer Prädiktor der Mortalität, der Hospitalisationsrate und der Exazerbationsrate. Weitere unabhängige Faktoren neben dem BODE-Index sind vor allem die körperliche Aktivität und kardiale Begleiterkrankungen. Auch nach den Ergebnissen der ECLIPSE-Studie waren die Ergebnisse mit dem BODE-Index sehr ähnlich wie nach Gruppierung anhand der FEV1 [1].

\section{Klinischer Endpunkt}

Neuere klinische Studien berücksichtigen den BODE-Index als Zielparameter. Man konnte medikamentös induzierte Verbesserungen in einer Größenordnung von 0,5 Punkten zeigen [92]. Auch Effekte einer pulmonalen Rehabilitation oder einer Inhalationsschulung ließen sich mit signifikanten Veränderungen des BODE-Index abbilden [93]. Nach Lungenvolumenreduktions- chirurgie ergab sich eine gute Korrelation des BODE-Index mit der Überlebensrate [94]. Bei Patienten mit schwerem Lungenemphysem wurden longitudinale Veränderungen des BODE-Index berechnet [95]. Verschlechterungen um mindestens einen Punkt waren zwar mit einer höheren Mortalität assoziiert, jedoch gab es insgesamt eine deutliche Überlappung der Überlebenskurven.

\section{Stärken und Schwächen}

Stärken und Schwächen des BODE-Index wurden in einer aktuellen Übersichtsarbeit zusammengefasst [26]. Der BODE-Index integriert unterschiedliche Facetten der COPD und ist hinsichtlich Mortalität und Exazerbationsrate seiner Einzelkomponente FEV1 überlegen. Vorteilhaft ist auch, dass er auf eindeutig zu erfassenden Parametern beruht. Für die Langzeit-Mortalität erscheint der BODE-Index weniger geeignet, da er für die Voraussage der Ein-Jahres-Mortalität optimiert wurde. Die Task Force der amerikanischen und europäischen Fachgesellschaften ATS/ ERS empfiehlt als Endpunkte pharmakologischer Studien neben der FEV1 diverse andere Parameter, vor allem Mortalität, Grad der Dyspnoe sowie die gesundheitsbezogene Lebensqualität [31]. Der BODE-Index macht deutlich, dass die COPD mehr ist als nur Atemwegsobstruktion. Für die Führung des individuellen Patienten ist der Index nicht geeignet. BODE-Index oder andere kombinierte Endpunkte müssen erst gründlich evaluiert werden, bevor sie in großem Maße in Therapiestudien eingesetzt werden.

\section{Aktuelle COPD-Studien}

\section{Referent: M. Pfeifer}

Auf der Internet-Plattform ClinicalTrials.gov sind derzeit 1113 Studien zur COPD verzeichnet. Schwerpunkte liegen in Europa und in den USA mit jeweils 550 bzw. 376 Studien. Neben klinischen Prüfungen zu neuen Substanzen und therapeutischen Targets werden Untersuchungen zum Phänotyp, zu genetischen Determinanten der COPD, zur Prävention und zu nicht-medikamentösen Therapiestrategien durchgeführt.

\section{COPD Gene Study}

Mit der COPD Gene Study soll die genetische Epidemiologie der COPD besser beschrieben werden [96]. Für die phänotypische Charakterisierung werden neben klinischen und physiologischen Parametern thorakale CT-Scans herangezogen. Ziel ist, Patienten mit COPD in Subgruppen aufzutrennen, die ätiologisch homogener sind. Zusätzlich sollen genetische Risikofaktoren der COPD und ihrer Subtypen in einer genomweiten Assoziationsstudie beschrieben werden. Erfasst werden sollen insgesamt rund 10500 Patienten, von denen bereits 9000 in das Projekt aufgenommen werden konnten. Die teilnehmenden 17 Zentren aus den USA werden durch ein administratives sowie durch ein Datenkoordinations-Zentrum unterstützt. Das Projekt wird von den National Institutes of Health $(\mathrm{NIH})$ finanziert.

Auf der Konferenz der amerikanischen Thorax-Gesellschaft ATS in 2010 wurden 23 Abstracts aus der COPD Gene Study präsentiert. Für die Zukunft wird eine Flut von Publikationen aus diesem Projekt erwartet.

\section{SPIROMICS}

Dieses Akronym bezeichnet die „Subpopulations and intermediate outcome measures in COPD study“. Auch hier geht es darum, Subpopulationen der COPD zu identifizieren. Zusätzlich sollen valide Surrogatmarker identifiziert werden, die sich als Ziel- 
parameter für klinische Studien eignen und Indikatoren für die Schwere der Erkrankung sind. An diesem NIH-finanzierten Projekt nehmen neun Universitäten aus den USA teil. Erhoben werden klinische und physiologische Parameter, serielle und dynamische CT-Scans sowie patientenrelevante Endpunkte. Zusätzlich sind umfangreiche Untersuchungen an diversen Biomaterialien (Blut, Urin, Sputum, Atemexhalat, BAL) geplant.

\section{ECLIPSE}

Das Projekt „Evaluation of COPD Longitudinally to Identify Predictive Surrogate Endpoints“ verläuft über drei Jahre und soll Parameter der Krankheitsprogression bei europäischen COPDPatienten definieren. Auch hier sollen Biomarker identifiziert werden, die sich als Surrogat-Endpunkte eignen. Neben 2180 COPD-Patienten werden zwei Vergleichskohorten mitgeführt, und zwar 343 Raucher und Ex-Raucher sowie 223 Personen, die noch nie geraucht haben. Studienteilnehmer werden über drei Jahre prospektiv beobachtet. Es sind acht Studienbesuche in halbjährlichen Abständen vorgesehen. Neben Spirometrien und Bodyplethysmografien werden auch IOS-Messungen erfolgen. Patienten füllen Fragebögen zu Symptomen, Dyspnoe, Fatigue, Lebensqualität und Depression aus. Vorgesehen sind außerdem Bildgebung (CT), ein 6-Minuten-Gehtest sowie die Messung von Sauerstoffsättigung und Körperzusammensetzung.

\section{STATSCOPE}

Dieses Präventions-Projekt hat zum Ziel, die Häufigkeit und Schwere von COPD-Exazerbationen mit Simvastatin zu verringern (Simvastatin Therapy for Moderate and Severe COPD). Dabei liegt der Fokus auf schwerer kranken Patienten, die eine Langzeitsauerstofftherapie erhalten oder im Jahr vor Studienbeginn entweder wegen einer COPD-Exazerbation hospitalisiert werden mussten oder systemische Antibiotika, systemische Kortikoide oder eine Notfallbehandlung erhalten hatten. Die Rationale, solchen Patienten Statine zu geben, basiert auf retrospektiven Analysen. Auch die STATSCOPE-Studie wird von den NIH finanziert.

\section{Studien zu eingeführten Therapien}

Ein in Großhansdorf basiertes Projekt soll überprüfen, ob inhalative Steroide abgesetzt werden können, wenn Patienten zwei potente langwirksame Bronchodilatatoren anwenden. Primärer Endpunkt ist die Zeit bis zur ersten mittelgradigen oder schweren Exazerbation der COPD innerhalb der 48-wöchigen Beobachtungszeit.

Die LOTT (Long-term Oxygen Treatment Trial) Studie prüft bei hypoxämischen COPD-Patienten einen möglichen Überlebensvorteil unter kontinuierlicher Sauerstofftherapie. In 14 US-amerikanischen Zentren wurden mehr als 3000 Patienten aufgenommen, die bis zu viereinhalb Jahre nachbeobachtet werden. Sie müssen bereit sein, über 24 Stunden kontinuierlich Sauerstoff anzuwenden. Erste Ergebnisse werden im Jahr 2012 erwartet.

Eine Studie der kanadischen Gesundheitsbehörde zur nächtlichen Sauerstofftherapie (CANOX) wendet sich an Patienten mit nächtlicher Desaturation, die nicht die Kriterien für eine Langzeitsauerstofftherapie erfüllen. Die Patienten erhalten nachts Sauerstoff und werden über drei Jahre nachbeobachtet. Analysiert werden sollen die Mortalität, die Kosteneffizienz und die Möglichkeit des Hinauszögerns einer kontinuierlichen Sauerstofftherapie.

COPD-Exazerbationen durch Antibiotika-Prophylaxe zu verhindern, ist Ziel der MACRO-Studie, die vom COPD Clinical Research Network durchgeführt wird. Über 12 Monate werden die Patien- ten kontinuierlich mit niedrig dosiertem Azithromycin bzw. mit Placebo behandelt. Andere klinische Prüfungen mit einer ähnlichen Ausrichtung verwenden inhalatives Levofloxacin oder andere neue inhalative Antibiotika.

\section{Neue Substanzen}

Bei der Pharmakotherapie liegt der Schwerpunkt auf der einmal täglichen Anwendung lang wirksamer Bronchodilatatoren als Mono- oder als Kombinationstherapie. Dabei geht es sowohl um LABAs (lang wirksame Beta-2-Sympathomimetika) als auch um LAMAs (lang wirksame Anticholinergika). Zur letztgenannten Gruppe gehört die Novartis-Substanz NVA237. Sie wird in einer Phase-III-Studie überprüft, an der auch deutsche Zentren teilnehmen. Glaxo Smith Kline entwickelt einen inhalativen Phosphodiesterase (PDE) 4-Hemmer, der sich derzeit in Phase I befindet.

\section{Nichtmedikamentöse Therapie-Strategien}

Neue bronchoskopische, interventionelle Verfahren haben unterschiedliche Ergebnisse mit teilweise nur marginalen Vorteilen erbracht. So wurden in der VENT-Studie nach Einsatz endobronchialer Ventile vermehrt Exazerbationen gesehen. Die Dekompression von Bronchien mit eingesetzten Spiralen scheint nur in wenigen Zentren zu funktionieren. Spiralen erzeugen häufig Sekretstau, sie können ihre Lokalisation verändern oder mit der Schleimhaut verwachsen, sodass man sie nicht mehr entfernen kann.

Die Selektion geeigneter Patienten zur interventionellen Volumenreduktion ist hier ganz besonders wichtig. Dabei könnte die Messung der kollateralen Ventilation sinnvoll sein. Ein entsprechendes Messgerät wird derzeit in Essen erprobt.

Die Patienten so zu schulen, dass sie ihre Fähigkeit zum Selbstmanagement steigern konnten, gelang in einem erfolgreichen Disease Management Programm [97]. Die kurze Intervention umfasste nur eine anderthalbstündige Schulung. Zusätzlich erhielten alle Patienten einen Aktionsplan für die Selbstbehandlung einer Exazerbation und es gab monatliche Telefonate mit dem Case Manager. Während eines Jahres waren Krankenhausaufenthalte bzw. Notfallbesuche in der Verumgruppe 28\% bzw. $27 \%$ seltener als in der Kontrollgruppe.

\section{GOLD Guideline in Diskussion \\ $\nabla$}

\section{Referent: C. Vogelmeier}

Die aktuelle Version der GOLD-Leitlinie zur COPD stammt aus dem Jahr 2009. Die Leitlinien-Gruppe diskutiert derzeit die Änderungen für die Version 2011.

\section{Parallelen zur Asthma-Leitlinie GINA}

Die Experten haben sich intensiv mit den Änderungen beschäftigt, die bei der GINA-Leitlinie zum Asthma bronchiale eingetreten sind [98]. Die klassische Definition des Asthma-Schweregrades als Basis der Therapie wurde im Jahr 2006 durch das Konzept der Asthmakontrolle ersetzt. Es beinhaltet unterschiedliche Komponenten, wie Symptome, Lungenfunktion, Exazerbationen und Notfallmedikation. Anlass für die Änderung waren die Ergebnisse der GOAL-Studie zur Step-up-Therapie mit Salmeterol und Fluticason [99]. Mit dem Asthmakontrolltest ACT steht ein einfaches Instrument zur Erhebung der Asthmakontrolle zur Verfügung [100]. Der Patient bewertet seine Situation in den letzten vier Wochen mit fünf Fragen, die mit jeweils fünf Punkten als 
bestem Wert beantwortet werden können. Es resultiert eine optimale Punktzahl von 25.

Das Konzept der Asthmakontrolle erscheint sinnvoll und logisch. Kritisch muss angemerkt werden, dass es noch nicht ausreichend validiert ist. Ein Zusammenhang zwischen dem gegenwärtigen Zustand und der Prognose des Patienten wurde in einer retrospektiven Analyse von fünf Therapiestudien aufgezeigt [101]. Nach Anwendung des Budesonid-Formoterol-Kombinationspräparates besserte sich die Asthmakontrolle und gleichzeitig ging die Rate der Exazerbationen innerhalb eines Jahres zurück. Diesbezüglich ist also das Paradigma erfüllt, dass die gegenwärtige Asthmakontrolle prognostisch relevant ist.

\section{Definition der COPD}

Zwischen den GOLD-Leitlinien aus 2006 und 2009 gibt es keinen Unterschied der COPD-Definition. Es handelt sich um eine reine Deskription. Erwähnt wird darin auch eine abnorme Entzündungsantwort auf schädliche Partikel oder Gase, wobei unklar bleibt, worin eine „abnorme“ inflammatorische Reaktion besteht.

In einer aktuellen Übersichtsarbeit wurden die systemischen Manifestationen der COPD und die Komorbiditäten zusammengefasst [102]. Darin wird deutlich, dass das bisherige „pulmozentrische Weltbild“ der Pneumologen den aktuellen Forschungsergebnissen zur COPD nicht mehr standhält.

Zunehmend im Fokus steht die reduzierte körperliche Aktivität, die mit einer verstärkten Entzündungsantwort und einer reduzierten Belastbarkeit von COPD-Patienten einhergeht $[60,103]$. Die günstigen Wirkungen körperlicher Aktivität bei diversen chronischen Krankheiten hängen möglicherweise mit dem Protein PGC-1 $\alpha$ (Peroxisome proliferator-activated receptor $\gamma$ coactivator $1 \alpha$ ) zusammen [104]. Seine Expression wird durch körperliche Bewegung induziert. Ein Mangel an PGC- $1 \alpha$ ist einerseits mit Muskelabbau assoziiert, andererseits werden diverse inflammatorische Markergene induziert, beispielsweise für IL-6 oder TNF- $\alpha$. Körperliche Aktivität hat also molekulare Auswirkungen und verändert die Expression bestimmter Gene.

\section{Lungenfunktionstestung}

Gegenwärtig wird zur Definition der COPD eine FEV1/FVC-Ratio $<0,7$ herangezogen. Bei Verwendung dieses fixen Verhältnisses besteht bei Personen über 60 Jahren das Risiko einer Überdiagnose [105]. Umgekehrt werden junge Personen möglicherweise nicht als COPD-krank erkannt. Als Alternative für einen Richtwert kommt die untere Normalwertgrenze infrage (LLN, lower limit of normal). Allerdings fehlen hierzu gut etablierte Sollwerte großer Populationen.

Für die Diagnose der COPD wird bisher ein Broncholysetest gefordert. Eine Verbesserung der FEV1 um mindestens $200 \mathrm{ml}$ und $12 \%$ gegenüber dem präbronchodilatatorischen Wert gilt als signifikante Reversibilität. In der UPLIFT-Kohorte waren von den Patienten im Stadium II rund 60\% und im Stadium IV rund 20\% reversibel [106]. Insofern stellt sich die Frage, ob die FEV1-Reversibilität ein sinnvolles Diagnose-Kriterium für die COPD ist. Die Briten haben dieses Kriterium in ihren NICE-Leitlinien von 2010 bereits modifiziert [107].

\section{Klassifizierung der Erkrankung}

Ähnlich wie bei den Asthma-Leitlinien gibt es Überlegungen, den Schweregrad der COPD nicht nur nach spirometrischen Ergebnissen einzuteilen. Vielmehr erscheint es sinnvoll, die aktuelle Änderung der Symptomatik einzubeziehen beispielsweise die Ver- besserung der Symptome und der körperlichen Belastbarkeit sowie einen insgesamt besseren Gesundheitszustand. Ein Test zum Erfassen der individuellen Symptomatik wurde in den letzten Jahren entwickelt (CAT, COPD Assessment Test) und validiert [108].

Zukünftig sollte die COPD mit einem mehr holistisch ausgerichteten Ansatz betrachtet werden.

\section{Abschließende Bemerkungen}

Die Tagung, deren Ergebnisse hier zusammengefasst werden, hat sich vorgenommen zu untersuchen, welche Fortschritte klinische Studien in der Diagnostik und Therapie der COPD erzielt haben. Neben den Ergebnissen standen auch die Studieninstrumente im Vordergrund. Ernüchtert musste festgestellt werden, dass auch robuste und häufig verwendete Parameter wie die Einsekundenkapazität $\left(\mathrm{FEV}_{1}\right)$ tückisch sind. Die Komplexität der Erkrankung, die unterschiedliche Subtypen ausprägt, entzieht sich einem eindimensionalen Verständnis.

In den Beiträgen werden einige aktuelle Tendenzen deutlich, die das Bild der COPD erheblich bereichert haben und die bei der Entwicklung zukünftiger Hypothesen und ihrer Verifikation helfen:

- Genetische Grundlagen und wesentliche Phänotypen der Erkrankung werden systematisch untersucht und als Stratifizierungsgrundlage entwickelt.

- Die Suche nach neuen, prognoserelevanten Surrogatparametern wird intensiviert. Dazu gehören neben Kompositendpunkten auch Biomarker.

- Die Bedeutung der spontanen körperlichen Aktivität und der Belastbarkeit der Patienten in der Beurteilung der Krankheitsprogression nimmt zu.

- Mit den neuen Instrumenten wird der Wert medikamentöser und nicht medikamentöser (z.B. Sauerstoff, maschinelle Atmungshilfe) Therapien neu evaluiert.

- Die Schwerpunkte in der Entwicklung von Pharmaka sind lang wirksame Broncholytika und neue antiinflammatorische Substanzen.

\section{Interessenkonflikt}

$\nabla$

In den letzten fünf Jahren haben die Referenten von Unternehmen oder Organisationen, die von den im Manuskript dargestellten Ergebnissen finanzielle Vor- oder Nachteile haben könnten, folgende Zuwendungen erhalten:

H. Worth, H. Magnussen, W. Randerath, A. Gillissen und J. Lorenz erhielten die Erstattung von Reisekosten oder Teilnahmegebühren für einen Kongress oder eine Fortbildungsveranstaltung.

H. Worth, A. Gillissen und J. Lorenz erhielten Honorare für Vorträge.

A. Gillissen erhielt Unterstützung für ein Forschungsvorhaben, finanzielle Zuwendungen für die Teilnahme an einer Studie, die Finanzierung von Mitarbeitern der Klinik sowie Honorar für Beratertätigkeit.

G. Steinkamp erhielt Erstattung von Reisekosten sowie Honorar für medizinisch-wissenschaftliches Publizieren.

C. Vogelmeier, M. Pfeifer, R. Bals, G. Schultze-Werninghaus und $\mathrm{H}$. Teschler geben keine Interessenkonflikte an.

Keiner der Autoren gab an, in irgendeiner Weise in den letzten fünf Jahren von der Tabak-Industrie gefordert worden zu sein oder sonstige finanzielle Interessenkonflikte zu sehen. 


\section{Institute}

1 Klinik für Pneumologie und Internistische Intensivmedizin, Klinikum Lüdenscheid

2 Pneumologie, Allergologie, Beatmungsmedizin, Universitätsklinikum des Saarlandes

3 Klinik für Lungen- und Bronchialmedizin, Klinikum Kassel

4 Pneumologisches Forschungsinstitut GmbH am Krankenhaus Großhansdorf

Klinik Donaustauf, Universität Regensburg

6 Klinik für Pneumologie und Allergologie, Krankenhaus Bethanien, Solingen

Emeritus, Berufsgenossenschaftliches Universitätsklinikum Bergmannsheil $\mathrm{GmbH}$

8 Medizinisch-wissenschaftliches Publizieren, Schwerin

9 Ruhrlandklinik, Westdeutsches Lungenzentrum, Universitätsklinikum Essen

${ }^{0}$ Klinik für Innere Medizin mit Schwerpunkt Pneumologie,

Universitätsklinikum Gießen und Marburg, Standort Marburg

1 Medizinische Klinik I, Klinikum Fürth

\section{Literatur}

1 Agusti A, Calverley PM, Celli B et al. Characterisation of COPD heterogeneity in the ECLIPSE cohort. Respir Res 2010; 11: 122

2 Hurst JR, Vestbo J, Anzueto A et al. Susceptibility to exacerbation in chronic obstructive pulmonary disease. N Engl J Med 2010; 363: $1128-1138$

3 The Alpha-Tocopherol, Beta Carotene Cancer Prevention Study Group. The Effect of Vitamin E and Beta Carotene on the Incidence of Lung Cancer and Other Cancers in Male Smokers. New England Journal of Medicine 1994; 330: 1029-1035

4 Omenn GS, Goodman GE, Thornquist MD et al. Effects of a Combination of Beta Carotene and Vitamin A on Lung Cancer and Cardiovascular Disease. New England Journal of Medicine 1996; 334: 1150-1155

5 Calverley PM, Anderson JA, Celli B et al. Salmeterol and fluticasone propionate and survival in chronic obstructive pulmonary disease. $\mathrm{N}$ Engl J Med 2007; 356: 775 - 789

6 McGarvey LP, John M, Anderson JA et al. Ascertainment of causespecific mortality in COPD: operations of the TORCH Clinical Endpoint Committee. Thorax 2007; 62: $411-415$

7 Sciurba FC, Ernst A, Herth FJF et al. A Randomized Study of Endobronchial Valves for Advanced Emphysema. New England Journal of Medicine 2010; 363: 1233 - 1244

8 Nishiyama K, Morimoto T, Furukawa Y et al. Chronic obstructive pulmonary disease - an independent risk factor for long-term cardiac and cardiovascular mortality in patients with ischemic heart disease. Int J Cardiol 2010; 143: 178-183

9 Barr RG, Bluemke DA, Ahmed FS et al. Percent emphysema, airflow obstruction, and impaired left ventricular filling. N Engl J Med 2010; 362: $217-227$

10 Watz H, Waschki B, Meyer T et al. Decreasing cardiac chamber sizes and associated heart dysfunction in COPD: role of hyperinflation. Chest 2010; 138: $32-38$

11 Selcuk H, Maden O, Selcuk MT et al. Documentation of impaired coronary blood flow in chronic obstructive pulmonary disease patients. Circ J 2010; 74: 346 - 352

12 Burgel PR, Paillasseur JL, Caillaud D et al. Clinical COPD phenotypes: a novel approach using principal component and cluster analyses. Eur Respir J 2010; 36: 531 - 539

13 Han MK, Agusti A, Calverley PM et al. Chronic obstructive pulmonary disease phenotypes: the future of COPD. Am J Respir Crit Care Med 2010; 182: $598-604$

14 Tashkin DP, Celli B, Kesten S et al. Long-term efficacy of tiotropium in relation to smoking status in the UPLIFT trial. Eur Respir J 2010; 35 : $287-294$

15 Troosters T, Celli B, Lystig $T$ et al. Tiotropium as a first maintenance drug in COPD: secondary analysis of the UPLIFT trial. Eur Respir J 2010; 36: $65-73$

16 Peters SP, Kunselman SJ, Icitovic $N$ et al. Tiotropium bromide step-up therapy for adults with uncontrolled asthma. N Engl J Med 2010; 363: $1715-1726$

17 Machado MC, Vollmer WM, Togeiro SM et al. CPAP and survival in moderate-to-severe obstructive sleep apnoea syndrome and hypoxaemic COPD. Eur Respir J 2010; 35: 132 - 137

18 Marin JM, Soriano JB, Carrizo SJ et al. Outcomes in patients with chronic obstructive pulmonary disease and obstructive sleep apnea: the overlap syndrome. Am J Respir Crit Care Med 2010; 182: 325 331
19 Tashkin DP, Celli B, Senn S et al. A 4-year trial of tiotropium in chronic obstructive pulmonary disease. N Engl J Med 2008; 359: 1543 - 1554

20 Vestbo J. The TORCH (towards a revolution in COPD health) survival study protocol. Eur Respir J 2004; 24: 206 - 210

21 Vestbo J, Anderson W, Coxson HO et al. Evaluation of COPD Longitudinally to Identify Predictive Surrogate End-points (ECLIPSE). Eur Respir J 2008; 31: $869-873$

22 Decramer M, Dekhuijzen PN, Troosters T et al. The Bronchitis Randomized On NAC Cost-Utility Study (BRONCUS): hypothesis and design. BRONCUS-trial Committee. Eur Respir J 2001; 17: 329 - 336

23 Calverley PM, Rabe KF, Goehring UM et al. Roflumilast in symptomatic chronic obstructive pulmonary disease: two randomised clinical trials. Lancet 2009; 374: 685 - 694

24 Fabbri LM, Calverley PM, Izquierdo-Alonso JL et al. Roflumilast in moderate-to-severe chronic obstructive pulmonary disease treated with longacting bronchodilators: two randomised clinical trials. Lancet 2009; 374: 695 - 703

25 Tashkin DP. The role of patient-centered outcomes in the course of chronic obstructive pulmonary disease: how long-term studies contribute to our understanding. Am J Med 2006; 119: 63- 72

26 Glaab T, Vogelmeier C, Buhl R. Outcome measures in chronic obstructive pulmonary disease (COPD): strengths and limitations. Respir Res 2010; 11: 79

27 Vogelmeier C, Buhl R, Criée CP et al. Leitlinie der Deutschen Atemwegsliga und der Deutschen Gesellschaft für Pneumologie und Beatmungsmedizin zur Diagnostik und Therapie von Patienten mit chronisch obstruktiver Bronchitis und Lungenemphysem (COPD). Pneumologie 2007; 61: e1 - e40

28 Enright P, Brusasco V. Counterpoint: should we abandon FEV/ FVC $<0.70$ to detect airway obstruction? Yes. Chest $2010 ; 138: 1040-$ 1042

29 Vandevoorde J, Verbanck S, Schuermans Det a. Obstructive and restrictive spirometric patterns: fixed cut-offs for FEV1/FEV6 and FEV6. Eur Respir J 2006; 27: 378-383

30 Swanney MP, Jensen RL, Crichton DA et al. FEV(6) is an acceptable surrogate for FVC in the spirometric diagnosis of airway obstruction and restriction. Am J Respir Crit Care Med 2000; 162: 917-919

31 Cazzola M, MacNee W, Martinez FJ et al. Outcomes for COPD pharmacological trials: from lung function to biomarkers. Eur Respir J 2008; 31: $416-469$

32 Taube C, Lehnigk B, Paasch K et al. Factor analysis of changes in dyspnea and lung function parameters after bronchodilation in chronic obstructive pulmonary disease. Am J Respir Crit Care Med 2000; 162: $216-220$

33 Stevenson NJ, Walker PP, Costello RW et al. Lung mechanics and dyspnea during exacerbations of chronic obstructive pulmonary disease. Am J Respir Crit Care Med 2005; 172: 1510-1516

34 Celli BR, Cote CG, Marin JM et al. The body-mass index, airflow obstruction, dyspnea, and exercise capacity index in chronic obstructive pulmonary disease. N Engl J Med 2004; 350: 1005 - 1012

35 O'Donnell DE. Dyspnea in advanced chronic obstructive pulmonary disease. J Heart Lung Transplant 1998; 17: 544-554

36 O'Donnell DE, Fluge T, Gerken F et al. Effects of tiotropium on lung hyperinflation, dyspnoea and exercise tolerance in COPD. Eur Respir J 2004; 23: $832-840$

37 Casanova C, Cote C, de Torres JP et al. Inspiratory-to-total lung capacity ratio predicts mortality in patients with chronic obstructive pulmonary disease. Am J Respir Crit Care Med 2005; 171: 591 - 597

38 Burrows B, Bloom JW, Traver GA et al. The course and prognosis of different forms of chronic airways obstruction in a sample from the general population. N Engl J Med 1987; 317: 1309-1314

39 Seemungal TA, Hurst JR, Wedzicha JA. Exacerbation rate, health status and mortality in COPD - a review of potential interventions. Int J Chron Obstruct Pulmon Dis 2009; 4: 203 - 223

40 Monto AS, Higgins MW, Ross HW. The Tecumseh study of respiratory illness. VIII. Acute infection in chronic respiratory disease and comparison groups. Am Rev Respir Dis 1975; 111: 27-36

41 Wedzicha JA, Calverley PM, Seemungal TA et al. The prevention of chronic obstructive pulmonary disease exacerbations by salmeterol/ fluticasone propionate or tiotropium bromide. Am J Respir Crit Care Med 2008; 177: 19-26

42 Soler-Cataluna JJ, Martinez-Garcia MA, Roman SP. Severe acute exacerbations and mortality in patients with chronic obstructive pulmonary disease. Thorax 2005; 60: 925 -931 
43 Anzueto A, Ferguson GT, Feldman G et al. Effect of fluticasone propionate/salmeterol (250/50) on COPD exacerbations and impact on patient outcomes. COPD 2009; 6: 320-329

44 Miravitlles M, Ferrer M, Pont A et al. Effect of exacerbations on quality of life in patients with chronic obstructive pulmonary disease: a 2 year follow up study. Thorax 2004; 59: 387-395

$45 \mathrm{Xu}$ W, Collet JP, Shapiro $S$ et al. Negative impacts of unreported COPD exacerbations on health-related quality of life at 1 year. Eur Respir J 2010; 35: 1022 - 1030

46 Eagan TM, Ueland T, Wagner PD et al. Systemic inflammatory markers in COPD: results from the Bergen COPD Cohort Study. Eur Respir J 2010; 35: $540-548$

47 Patel IS, Seemungal TA, Wilks $M$ et al. Relationship between bacterial colonisation and the frequency, character, and severity of COPD exacerbations. Thorax 2002; 57: 759- 764

48 Miravitlles M, Marin A, Monso E et al. Efficacy of moxifloxacin in the treatment of bronchial colonisation in COPD. Eur Respir J 2009; 34: $1066-1071$

49 Sethi S, Jones $P W$, Theron MS et al. Pulsed moxifloxacin for the prevention of exacerbations of chronic obstructive pulmonary disease: a randomized controlled trial. Respir Res 2010; 11: 10

50 Suissa S, Ernst P, Vandemheen KL, Aaron SD. Methodological issues in therapeutic trials of COPD. Eur Respir J 2008; 31: 927-933

51 Calverley PM, Stockley RA, Seemungal TA et al. Reported Pneumonia in COPD: Findings From the INSPIRE Study. Chest 2010; 139: 505-512

52 Chang ET. Tiotropium in chronic obstructive pulmonary disease. $\mathrm{N}$ Engl J Med 2009; 360: 185

53 Singh S, Furberg CD, Loke YK. Tiotropium in chronic obstructive pulmonary disease. N Engl J Med 2009; 360: 186

54 Pedone C, Incalzi RA. Tiotropium in chronic obstructive pulmonary disease. N Engl J Med 2009; 360: 185

55 Wegner RE, Jorres RA, Kirsten DK et al. Factor analysis of exercise capacity, dyspnoea ratings and lung function in patients with severe COPD. Eur Respir J 1994; 7: 725 - 729

56 ATS Committee on Proficiency Standards for Clinical Pulmonary Function Laboratories. ATS statement: guidelines for the six-minute walk test. Am J Respir Crit Care Med 2002; 166: 111 - 117

57 Enright PL, Sherrill DL. Reference equations for the six-minute walk in healthy adults. Am J Respir Crit Care Med 1998; 158: 1384 - 1387

58 Troosters T, Gosselink R, Decramer M. Six minute walking distance in healthy elderly subjects. Eur Respir J 1999; 14: 270 - 274

59 Cote CG, Casanova C, Marin JM et al. Validation and comparison of reference equations for the 6-min walk distance test. Eur Respir J 2008; 31: $571-578$

60 Watz H, Waschki B, Meyer T et al. Physical activity in patients with chronic obstructive pulmonary disease. Eur Respir J 2009; 33: 262 272

61 Spruit MA, Watkins ML, Edwards LD et al. Determinants of poor 6-min walking distance in patients with COPD: the ECLIPSE cohort. Respir Med 2010; 104: 849-857

62 von Leuopoldt A, Taube K, Schubert-Heukeshoven S et al. Distractive auditory stimuli reduce the unpleasantness of dyspnea during exercise in patients with COPD. Chest 2007; 132: $1506-1512$

63 Wewel AR, Gellermann I, Schwertfeger I et al. Intervention by phone calls raises domiciliary activity and exercise capacity in patients with severe COPD. Respir Med 2008; 102: 20-26

64 Behnke $M$, Wewel AR, Kirsten $D$ et al. Exercise training raises daily activity stronger than predicted from exercise capacity in patients with COPD. Respir Med 2005; 99: 711-717

65 Young J, Fry-Smith A, Hyde C. Lung volume reduction surgery (LVRS) for chronic obstructive pulmonary disease (COPD) with underlying severe emphysema. Thorax 1999; 54: 779-789

66 Hay JG, Stone P, Carter J et al. Bronchodilator reversibility, exercise performance and breathlessness in stable chronic obstructive pulmonary disease. Eur Respir J 1992; 5: 659-664

67 Leach RM, Davidson AC, Chinn S et al. Portable liquid oxygen and exercise ability in severe respiratory disability. Thorax 1992; 47: 781 789

68 Puhan MA, Mador MJ, Held U et al. Interpretation of treatment changes in 6-minute walk distance in patients with COPD. Eur Respir J 2008; 32: 637-643

69 Kirsten DK, Taube C, Lehnigk B et al. Exercise training improves recovery in patients with COPD after an acute exacerbation. Respir Med 1998; 92: $1191-1198$
70 Behnke M, Taube C, Kirsten D et al. Home-based exercise is capable of preserving hospital-based improvements in severe chronic obstructive pulmonary disease. Respir Med 2000; 94: 1184-1191

71 Troosters T, Probst VS, Crul T et al. Resistance training prevents deterioration in quadriceps muscle function during acute exacerbations of chronic obstructive pulmonary disease. Am J Respir Crit Care Med 2010; 181: 1072 - 1077

72 Thompson PD, Buchner D, Pina IL et al. Exercise and physical activity in the prevention and treatment of atherosclerotic cardiovascular disease: a statement from the Council on Clinical Cardiology (Subcommittee on Exercise, Rehabilitation, and Prevention) and the Council on Nutrition, Physical Activity, and Metabolism (Subcommittee on Physical Activity). Circulation 2003; 107: 3109- 3116

73 Lollgen H, Bockenhoff A, Knapp G. Physical activity and all-cause mortality: an updated meta-analysis with different intensity categories. Int J Sports Med 2009; 30: 213-224

74 Troosters T, Sciurba F, Battaglia $S$ et al. Physical inactivity in patients with COPD, a controlled multi-center pilot-study. Respir Med 2010 104: $1005-1011$

75 Hill K, Dolmage TE, Woon L et al. Measurement properties of the SenseWear armband in adults with chronic obstructive pulmonary disease. Thorax 2010; 65: 486-491

76 Hersh CP, DeMeo DL, Silverman EK. National Emphysema Treatment Trial state of the art: genetics of emphysema. Proc Am Thorac Soc 2008; 5: 486- 493

77 Dahl M, Tybjaerg-Hansen A, Lange P et al. Change in lung function and morbidity from chronic obstructive pulmonary disease in alpha1-antitrypsin MZ heterozygotes: A longitudinal study of the general population. Ann Intern Med 2002; 136: 270 - 279

78 Hunninghake GM, Cho MH, Tesfaigzi Y et al. MMP12, lung function, and COPD in high-risk populations. N Engl J Med 2009; 361: 2599 2608

79 Sin $D D$, Vestbo J. Biomarkers in chronic obstructive pulmonary disease. Proc Am Thorac Soc 2009; 6: 543-545

80 Dahl M, Tybjaerg-Hansen A, Vestbo J et al. Elevated plasma fibrinogen associated with reduced pulmonary function and increased risk of chronic obstructive pulmonary disease. Am J Respir Crit Care Med 2001; $164: 1008-1011$

81 de Torres JP, Cote CG, Lopez MV et al. Sex differences in mortality in patients with COPD. Eur Respir J 2009; 33: 528-535

82 Dahl M, Vestbo J, Zacho J et al. C reactive protein and chronic obstructive pulmonary disease: a Mendelian randomisation approach. Thorax 2010; 66: 197-204

83 Sin DD, Leung R, Gan WQ et al. Circulating surfactant protein D as a potential lung-specific biomarker of health outcomes in COPD: a pilot study. BMC Pulm Med 2007; 7: 13

84 Lomas DA, Silverman EK, Edwards LD et al. Evaluation of serum CC-16 as a biomarker for COPD in the ECLIPSE cohort. Thorax 2008; 63 : $1058-1063$

85 Alford SK, van Beek EJ, McLennan G et al. Heterogeneity of pulmonary perfusion as a mechanistic image-based phenotype in emphysema susceptible smokers. Proc Natl Acad Sci U S A 2010; 107: 7485-7490

86 Jones PW, Agusti AG. Outcomes and markers in the assessment of chronic obstructive pulmonary disease. Eur Respir J 2006; 27: 822 832

87 Sankaranarayanan V, Ziedalski T, Gould MK. Predicting outcomes in chronic obstructive pulmonary disease. N Engl J Med 2004; 350: $2308-2310$

88 Soler-Cataluna JJ, Martinez-Garcia MA, Sanchez LS et al. Severe exacerbations and BODE index: two independent risk factors for death in male COPD patients. Respir Med 2009; 103: 692 - 699

89 Lopez-Campos JL, Cejudo P, Marquez E et al. Modified BODE indexes: Agreement between multidimensional prognostic systems based on oxygen uptake. Int J Chron Obstruct Pulmon Dis 2010; 5: 133-140

90 Puhan MA, Garcia-Aymerich J, Frey M et al. Expansion of the prognostic assessment of patients with chronic obstructive pulmonary disease: the updated BODE index and the ADO index. Lancet 2009; 374: 704-711

91 Esteban C, Quintana JM, Moraza J et al. BODE-Index vs HADO-score in chronic obstructive pulmonary disease: Which one to use in general practice? BMC Med 2010; 8: 28

92 Dahl R, Chung KF, Buhl R et al. Efficacy of a new once-daily long-acting inhaled beta2-agonist indacaterol versus twice-daily formoterol in COPD. Thorax 2010; 65: 473-479 
93 Cote CG, Celli BR. Pulmonary rehabilitation and the BODE index in COPD. Eur Respir J 2005; 26: 630-636

94 Imfeld S, Bloch KE, Weder W et al. The BODE index after lung volume reduction surgery correlates with survival. Chest 2006; 129: 873878

95 Martinez FJ, Han MK, Andrei AC et al. Longitudinal change in the BODE index predicts mortality in severe emphysema. Am J Respir Crit Care Med 2008; 178: 491 - 499

96 Regan EA, Hokanson JE, Murphy JR et al. Genetic epidemiology of COPD (COPDGene) study design. COPD 2010; 7: $32-43$

97 Rice KL, Dewan N, Bloomfield HE et al. Disease management program for chronic obstructive pulmonary disease: a randomized controlled trial. Am J Respir Crit Care Med 2010; 182: 890 - 896

98 Bateman ED, GINA executive committee. Global strategy for asthma management and prevention. www.ginasthma.org 2009

99 Bateman ED, Boushey HA, Bousquet J et al. Can guideline-defined asthma control be achieved? The Gaining Optimal Asthma ControL study. Am J Respir Crit Care Med 2004; 170: 836-844

100 Nathan RA, Sorkness CA, Kosinski $M$ et al. Development of the asthma control test: a survey for assessing asthma control. J Allergy Clin Immunol 2004; 113: 59-65
101 Bateman ED, Reddel HK, Eriksson G et al. Overall asthma control: the relationship between current control and future risk. J Allergy Clin Immunol 2010; 125: 600-608

102 Barnes PJ, Celli BR. Systemic manifestations and comorbidities of COPD. Eur Respir J 2009; 33: 1165-1185

103 Watz H, Waschki B, Boehme C et al. Extrapulmonary effects of chronic obstructive pulmonary disease on physical activity: a cross-sectional study. Am J Respir Crit Care Med 2008; 177: 743-751

104 Handschin C, Spiegelman BM. The role of exercise and PGC1alpha in inflammation and chronic disease. Nature 2008; 454: 463-469

105 Mannino DM, Sonia BA, Vollmer WM. Chronic obstructive pulmonary disease in the older adult: what defines abnormal lung function? Thorax 2007; 62: 237-241

106 Tashkin DP, Celli B, Decramer $M$ et al. Bronchodilator responsiveness in patients with COPD. Eur Respir J 2008; 31: 742 - 750

107 National Clinical Guideline Centre. Chronic obstructive pulmonary disease: Management of chronic obstructive pulmonary disease in adults in primary and secondary care. London: National Clinical Guideline Centre; 2010

108 Jones PW, Harding G, Berry P et al. Development and first validation of the COPD Assessment Test. Eur Respir J 2009; 34: 648 - 654 